\title{
Biomechanical Measurement of Rabbit Cornea by a Modified Scheimpflug Device
}

\author{
Bo Zhang, ${ }^{1}$ Jianjun Gu, ${ }^{1}$ Xiaoxiao Zhang, ${ }^{1}$ Bin Yang, ${ }^{1}$ Zheng Wang, ${ }^{2}$ and Danying Zheng ${ }^{1}$ \\ ${ }^{1}$ State Key Lab of Ophthalmology, Zhongshan Ophthalmic Center, Sun Yat-Sen University, Guangzhou 510060, China \\ ${ }^{2}$ Guangzhou Aier Eye Hospital, Guangzhou 510288, China \\ Correspondence should be addressed to Zheng Wang; gzstwang@gmail.com and Danying Zheng; zhengdyy@163.com
}

Received 25 March 2016; Accepted 15 May 2016

Academic Editor: George M. Saleh

Copyright (c) 2016 Bo Zhang et al. This is an open access article distributed under the Creative Commons Attribution License, which permits unrestricted use, distribution, and reproduction in any medium, provided the original work is properly cited.

\begin{abstract}
Purpose. To explore the probability and variation in biomechanical measurements of rabbit cornea by a modified Scheimpflug device. Methods. A modified Scheimpflug device was developed by imaging anterior segment of the model imitating the intact eye at various posterior pressures. The eight isolated rabbit corneas were mounted on the Barron artificial chamber and images of the anterior segment were taken at posterior pressures of $15,30,45,60$, and $75 \mathrm{mmHg}$ by the device. The repeatability and reliability of the parameters including CCT, ACD, ACV, and CV were evaluated at each posterior pressure. All the variations of the parameters at the different posterior pressures were calculated. Results. All parameters showed good intraobserver reliability (Cronbach's alpha; intraclass correlation coefficient, $\alpha$, ICC > 0.96) and repeatability in the modified Scheimpflug device. With the increase of posterior pressures, the ratio of CCT decreased linearly and the bulk modulus gradually reduced to a platform. The increase of ACD was almost linear with the posterior pressures elevated. Conclusions. The modified Scheimpflug device was a valuable tool to investigate the biomechanics of the cornea. The posterior pressure $15-75 \mathrm{mmHg}$ range produced small viscoelastic deformations and nearly linear pressure-deformation response in the rabbit cornea.
\end{abstract}

\section{Introduction}

A comprehensive understanding of biomechanical properties of the cornea is crucial for a wide range of clinical applications such as keratoconus, tonometry, and refractive surgeries [13]. Corneal biomechanics involves the complex interaction between its lamellar collagen structure and the charged, hydrated proteoglycan gel. Many studies have indicated that the stress-strain response in mammalian corneas is typical for anisotropic collagenous tissues [4-6]. Strip extensometry and inflation tests were still the two important methods in biomechanical studies. The former was relatively simple and had low cost but involved three inherent deficiencies and had less reliability: a strip specimen is originally part of the cornea, its curvature flattens, and the back analysis usually considers the effect of the central cornea thickness and ignores the fact of the natural increase in cornea thickness away from the center [7]. The inflation tests were executed by altering the intraocular pressure (IOP) and measuring the apex displacement or central corneal tangential elongation on the intact eyes or corneal rigs, and then biomechanical parameters were calculated by mathematical analysis. Tissue markers on the epithelial and endothelial surfaces such as mercury droplets, adhesive tape marker, or dark and light contrasting regions of the cornea provided by graphite powder combined with digital cameras were usually needed in the inflation tests; however, these methods could disturb the cornea and had limited metrical parameters [8-10].

Pentacam (Oculus, Germany) using Scheimpflug photography as a basis has become a popular clinical device for measuring and characterizing the anterior segment. Many studies have indicated good repeatability and reproducibility in the measurements of topographic corneal thickness, anterior and posterior corneal curvatures, anterior chamber depth, and angle and corneal aberrations [11-19]. With the update of the software version, it also provides parameters such as corneal volume and keratometric power difference, which offer new and technical analyses of the cornea $[20,21]$. At 
present, Pentacam has been used in the range of study types including chronic applications over longer periods as well as dose range studies investigating a no-effect level with respect to lens changes in animal models. Rats, rabbits, dogs, cats, and monkeys all have ocular dimensions, which allow recording of optical sections of their eyes by Pentacam in a reproducible way [22]. The use of animal corneas as approximate models to human corneas in mechanical property characterization studies had been necessary because of the difficulties in obtaining enough human donor corneas.

In this study, we investigated the biomechanics of intact corneas of rabbits subjected to altering of posterior inflation pressures by a modified Scheimpflug device and evaluated the probability of this method.

\section{Materials and Methods}

2.1. Specimen Preparation. Eight fresh rabbit eyes from New Zealand white rabbits $(2-3 \mathrm{Kg}$, female) were obtained. A central disk including the corneal button and a $3 \mathrm{~mm}$ scleral ring was removed with a pair of curved scissors and this was followed by the removal of the iris, lens, and ciliary body under microscope within 1 hour of eye enucleation. Prior to specimen preparation the central corneal thickness was measured using a pachymeter (DGH1000, DGH Technologies, Exton, PA). The average value and standard deviation were $397.3 \pm 13.7 \mu \mathrm{m}$. The epithelium of the cornea was kept complete to lessen the effect of hydration in the procedure.

2.2. Inflation Tests. The corneas were mounted onto the artificial pressure chamber (K20-2126, Katena, USA). The pressure chamber was filled with saline solution and connected to a small reservoir, whose vertical movement represented the change of intraocular pressure. The actual pressure in the chamber was measured using a differential pressure transducer (YP101, Xinhang Co., China) and the measurements were recorded automatically in Bio-Signal Acquisition System (Chengdu TME Technology Co., China). The whole apparatus was fixed by two clamps between the two pillars of the Pentacam and moved vertically to attain the appropriate position. The maximum pressure applied was $75 \mathrm{mmHg}$, which was well above the normal physiological level. Three cycles of loading and unloading up to $75 \mathrm{mmHg}$ at intervals of 5 minutes were applied to recondition and stabilize its behavior of the cornea.

2.3. Experimental Protocol. The posterior inflation pressure was varied stepwise from $15 \mathrm{mmHg}$ to $75 \mathrm{mmHg}$ and the images were taken at $15,30,45,60$, and $75 \mathrm{mmHg}$. The pressure was always increased with the speed of $15 \mathrm{mmHg} / \mathrm{min}$. All images were taken in 5 minutes after the step increase in pressure to allow enough time for the equilibration of cornea creep. The surface of cornea was drenched using the solution (Dextran40 Sodium Chloride) in 10 seconds before taking images. The examiner adjusted the joystick until perfect alignment was shown, and then, the system automatically took images of the cornea within 2 -second period. Only those scans that registered as "OK" were included. Three consecutive scans were captured for intraobserver reliability analysis. The same procedures were repeated on a different pressure level.

2.4. Statistical Analysis. Statistical analysis was performed using SPSS software (version 19.0, SPSS, Inc., Chicago, IL, USA). The intraobserver reliability of all parameters was tested using Cronbach's alpha test $(\alpha)$ and the intraclass correlation coefficient (ICC). The intraobserver repeatability for each corneal parameter was assessed by the statistical parameters: the within-subject standard deviation, intraobserver precision, and intraobserver repeatability as previously reported [14]. All the data were tested for normality using the Kolmogorov-Smirnov test. The level of significance was set at 0.05 .

\section{Results}

3.1. Intraobserver Repeatability and Reliability of the Measurements for the Parameters. Like human screening by Pentacam, most parameters such as corneal curvatures, corneal thickness (CCT), anterior chamber depth (ACD), corneal elevations, anterior chamber volume (ACV), and corneal volume $(\mathrm{CV})$ were acquired by the modified Scheimpflug device. With the increase of the inflation pressure, all parameters varied without the loss of high quality of the images, even in high pressure of $75 \mathrm{mmHg}$.

The modified Scheimpflug device was shown to be highly reliable in the measurements of the parameters such as central corneal thickness, anterior chamber depth, anterior chamber, and corneal volumes $(\alpha>0.98$; ICCs $>0.96)$. With the varying of posterior pressure, the reliabilities of the measurements of the parameters did not change significantly $(p>$ 0.05 , Table 1$)$. High repeatabilities of the measurements of the parameters were also shown and did not alter significantly with the increase of posterior pressures $(p>0.05)$.

3.2. The Measurements of Corneal Thickness. The corneal thickness at posterior pressure of $15 \mathrm{mmHg}$ which is appropriately consistent with normal intraocular pressure of rabbit was viewed as the initial number of CCT. With a rise in posterior pressure, the average corneal thickness diminished. The changes of CCT to the primary corneal thickness at pressures were calculated as $\left(\mathrm{CCT}_{15 \mathrm{mmHg}}-\right.$ $\left.\mathrm{CCT}_{\text {posterior pressure }}\right) / \mathrm{CCT}_{15 \mathrm{mmHg}}$, and the ratios were shown linearly with the increase of posterior pressure (Figure 1).

3.3. Corneal Volume Measurements and Modulus of Volumetric Strain. Pachymetry software in the device can calculate the corneal volume within different range around the central cornea. Figure 2 shows the corneal volumes within $10 \mathrm{~mm}$ circle decrease with the increase of posterior pressures and the ratios of the decrease of the corneal volume are almost linear. In the inflation test, the stress and volumetric strain values derived from the pressure-deformation experimental results could be used to determine the variation of the bulk modulus with applied posterior pressure. If the cornea at posterior pressure of $15 \mathrm{mmHg}$ was defined as normal primary 
TABLE 1: Summary of intraobserver repeatability and reliability for the parameters.

\begin{tabular}{lcccccc}
\hline Parameters & $\begin{array}{c}\text { Posterior } \\
\text { pressure } \\
(\mathrm{mmHg})\end{array}$ & Sw & Pr & Rep & C alpha & ICC \\
\hline \multirow{4}{*}{ CCT } & 15 & 0.972 & 1.904 & 2.691 & 1.000 & 1.000 \\
& 30 & 1.028 & 2.014 & 2.847 & 1.000 & 1.000 \\
& 45 & 1.374 & 2.694 & 3.807 & 1.000 & 0.999 \\
& 60 & 1.460 & 2.863 & 4.046 & 1.000 & 0.999 \\
ACD & 75 & 1.713 & 3.357 & 4.744 & 1.000 & 0.999 \\
\hline \multirow{4}{*}{ ACV } & 15 & 0.032 & 0.062 & 0.088 & 0.998 & 0.994 \\
& 30 & 0.032 & 0.062 & 0.088 & 0.998 & 0.995 \\
& 45 & 0.032 & 0.062 & 0.088 & 0.998 & 0.996 \\
& 60 & 0.032 & 0.062 & 0.088 & 0.999 & 0.997 \\
& 75 & 0.032 & 0.062 & 0.088 & 0.999 & 0.998 \\
\hline & 15 & 2.402 & 4.709 & 6.655 & 0.998 & 0.993 \\
& 30 & 5.911 & 11.586 & 16.374 & 0.988 & 0.967 \\
& 45 & 2.448 & 4.799 & 6.782 & 0.998 & 0.995 \\
& 60 & 3.141 & 6.157 & 8.701 & 0.997 & 0.994 \\
& 75 & 6.011 & 11.782 & 16.651 & 0.994 & 0.976 \\
\hline \multirow{4}{*}{ CV } & 15 & 0.138 & 0.270 & 0.382 & 1.000 & 0.999 \\
& 30 & 0.118 & 0.232 & 0.328 & 1.000 & 1.000 \\
& 45 & 0.276 & 0.540 & 0.764 & 0.999 & 0.997 \\
& 60 & 0.138 & 0.270 & 0.382 & 1.000 & 0.999 \\
& 75 & 0.443 & 0.868 & 1.226 & 0.997 & 0.988 \\
\hline
\end{tabular}

Sw: within-subject standard deviation; Pr: precision; Rep: repeatability; ICC: intraclass correlation coefficient; CCT: central cornea thickness; ACV: anterior chamber volume; ACD: anterior chamber depth; CV: corneal volume.

condition, the average bulk modulus was 0.25 megapascals (Mpa) at the posterior pressure of $30 \mathrm{mmHg}$ and decreased to $0.11 \mathrm{Mpa}$ at $75 \mathrm{mmHg}$. Beyond the pressure $45 \mathrm{mmHg}$, the modulus was almost attainted at the platform (Figure 3).

3.4. The Measurements of Anterior Chamber. The rabbit corneas were mounted to the artificial chamber for simulating the intact eyeballs. The images of anterior chamber were scanned well by the modified device. With the increase of posterior pressures, both ACV and ACD gradually elevated and the change of ACD was almost linear (Figure 4).

\section{Discussion}

The present study employed a new modified Scheimpflug device and executed the inflation test to examine the rabbit corneal biomechanics. In the experiment, we mounted the rabbit corneas to the artificial chamber. The base of the artificial chamber was modified by black tape to simulate the iris structure. The light reflex reduced greatly and the back surface of the cornea was shown obviously in the scan of the modified device and high quality of the images of anterior segment was acquired. The objective of this study was to evaluate the intraobserver repeatability and reliability of

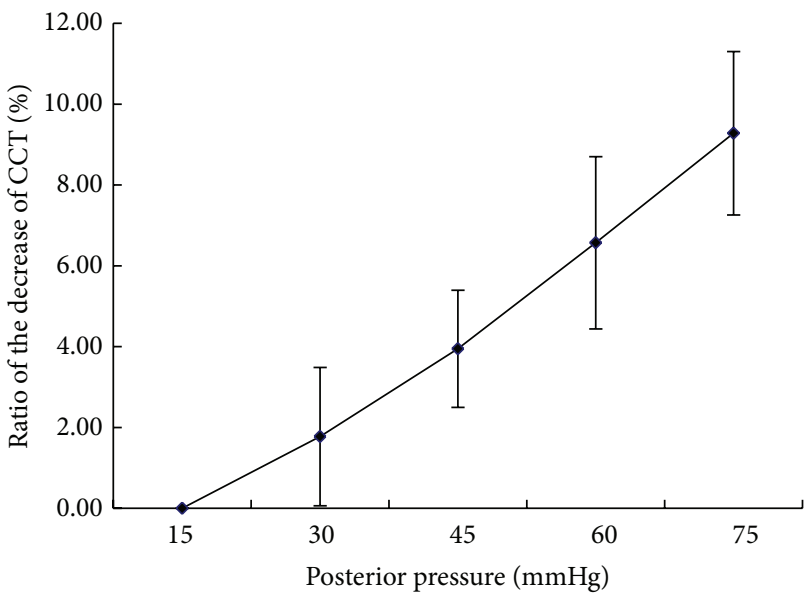

FIgURE 1: The change of central corneal thickness (CCT) of rabbit eyes with the increase of posterior pressure. The ratios of the decrease of CCT were calculated as $\left(\mathrm{CCT}_{15 \mathrm{mmHg}}-\right.$ $\left.\mathrm{CCT}_{\text {posterior pressure }}\right) / \mathrm{CCT}_{15 \mathrm{mmHg}}$, where $\mathrm{CCT}_{15 \mathrm{mmHg}}$ was viewed as the initial thickness of the cornea. The ratios were shown linearly with the increase of posterior pressure $\left(R^{2}=0.9928\right)$.

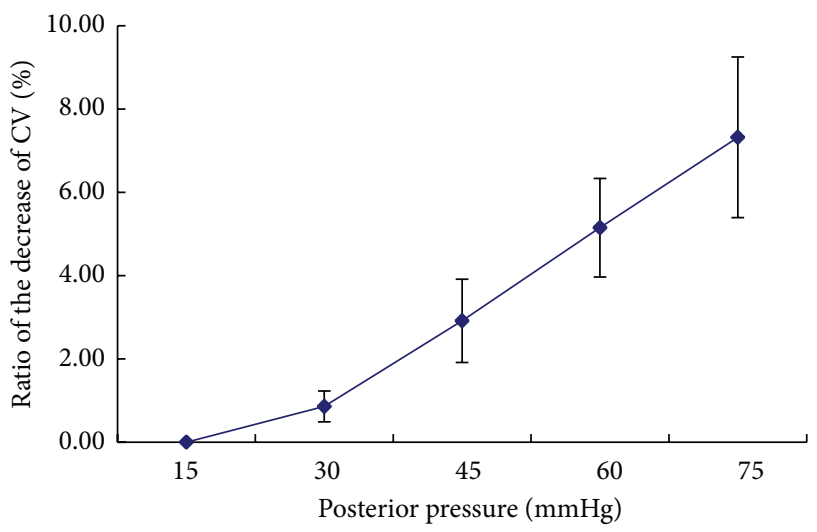

FIGURE 2: The change of rabbit corneal volume (CV) with the increase of posterior pressure. The ratios of the decrease of $\mathrm{CV}$ were calculated as $\left(\mathrm{CV}_{15 \mathrm{mmHg}}-\mathrm{CV}_{\text {posterior pressure }}\right) / \mathrm{CV}_{15 \mathrm{mmHg}}$. The ratios were shown linearly with the increase of posterior pressure $\left(R^{2}=0.98\right)$.

the modified device. In general, a value of 0.70 is considered satisfactory and in clinical applications a value of 0.90 is required. For the ICC, a value above 0.75 indicates good reliability, but most clinical applications require a value of at least 0.90 [13]. In the experiment, the values of ICC and $\alpha$ were almost 1.0 and excellent results even higher than those in human measurements as reported were shown in repeatability analysis [13]. There were two reasons to account for the facts: firstly, the device kept stable without any factors affecting the measurement such as eye movement or blink during the scan. Secondly, several studies reported that the creep was small for the normally hydrated and the swollen corneas and lasted between 30 and 70 seconds [23], so the posterior pressures continued for 5 minutes and ensured the equilibration of corneal creep without effects on 


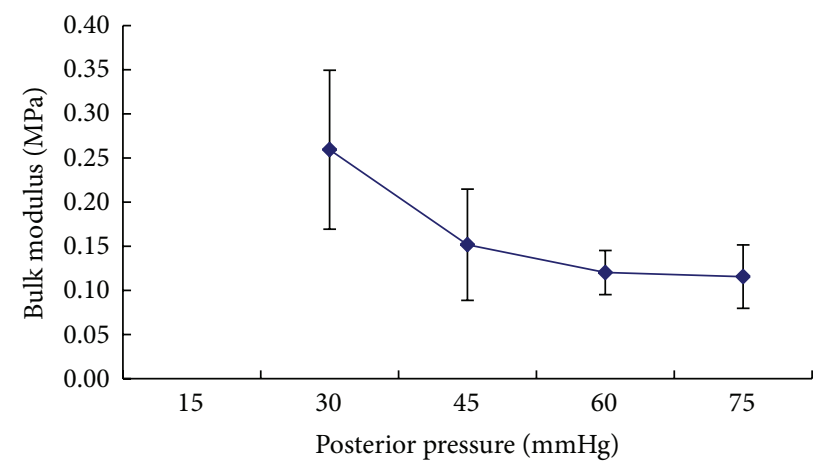

FIGURE 3: The change of bulk modulus of rabbit corneas with the increase of posterior pressure. The cornea at posterior pressure of $15 \mathrm{mmHg}$ was defined as primary condition $\left(P_{0}\right)$. The bulk modulus was calculated as $\Delta P / \Delta V$, where $\Delta P=\left(P-P_{0}\right), \Delta V=\left(\mathrm{CV}_{15 \mathrm{mmHg}}-\right.$ $\left.\mathrm{CV}_{\text {posterior pressure }}\right) / \mathrm{CV}_{15 \mathrm{mmHg}}$.

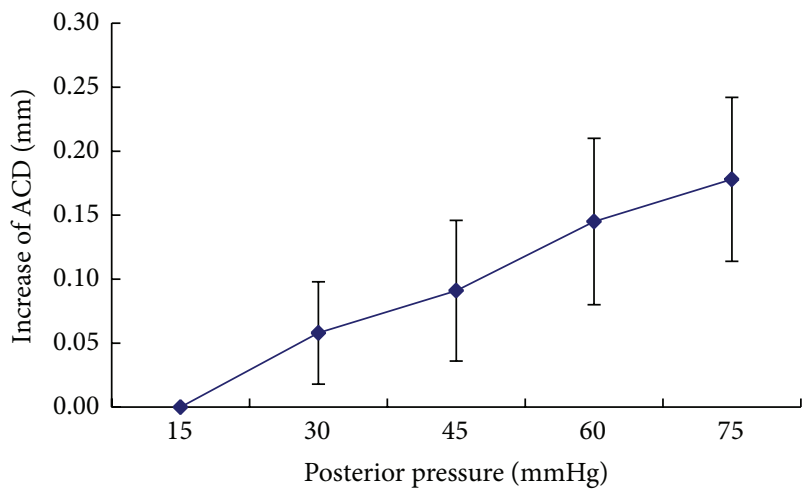

FIGURE 4: The variation of anterior chamber depth (ACD) of rabbit eyes with the increase of posterior pressure. The increase of ACD was calculated as $\left(\mathrm{ACD}_{\text {posterior pressure }}-\mathrm{ACD}_{15 \mathrm{mmHg}}\right)$. With the increase of posterior pressures, ACD gradually elevated and the change of ACD was almost linear $\left(R^{2}=0.9913\right)$.

the measurements of the cornea in the study. Our results indicated that the modified device had good reliability and repeatability in the measurements of the parameters, even still kept stable with the increase of posterior pressures. The modified device should be taking advantage of measuring the corneal biomechanics.

Until now corneal biomechanics has been challenging in the ophthalmic field because of difficulties of in vivo accurate measurements. Although two noncontact tonometers (Ocular Response Analyzer and CorVis ST) are currently commercially available to study corneal biomechanics, there are still obvious drawbacks in the two devices. In the former, whether the corneal resistance factor and corneal hysteresis accurately represent corneal biomechanics is questionable, even some studies have found that there is no direct relationship between the modulus of elasticity and the corneal hysteresis and this suggests that conclusions from studies using the ORA should be considered cautiously [24, 25]. In the latter, the Corvis ST does not provide conventional biomechanical parameters such as the elastic modulus and the stiffness. Furthermore, the area of analysis with the two devices is only limited to approximately the central $3.0 \mathrm{~mm}$ of the cornea, which does not represent all of the corneal biomechanics $[25,26]$. The inflation test is still a valuable method combined with shell theory to calculate stress, strain, and elastic modulus, which ensures the integrity of the corneal tissue in almost normal physiological condition.

Some studies have proved that corneal hydration affects the properties of corneal biomechanics and corneal thickness rises linearly with the increase of corneal hydration [27]. In the study, we kept the intact epithelium and minimize the evaporation on corneal surface drenched using the solution (Dextran40 Sodium Chloride) and the corneal hydration almost maintained normally in the procedure to minimize its effects on the properties of corneal biomechanics. In biomechanical studies, the corneal strain behaviors and corneal apex displacement were usually investigated. One study found that the human cornea showed a negligible extensibility and the rabbit tissue underwent a 9\% strain with a curvilinear relationship between stress and strain under low pressures and the relationship was linear at higher pressures [28]. Our data showed the linear relationship between posterior pressures and ACD increase similar with apex displacement beyond the normal physiologic IOP and they were consistent with previous reports.

In this study, we found that the corneal thickness decreased linearly with increasing intraocular pressure and the corneal thickness decreased by $9.28 \pm 2.02 \%$ for a pressure increase from $15 \mathrm{mmHg}$ to $75 \mathrm{mmHg}$, which was in good agreement with the results of Hennighausen et al. [23]. According to the incompressibility assumption, corneal volumetric considerations reveal that the relative change in stromal thickness would have been twice the tangential strain of the stroma and the reduction of the corneal thickness is generally viewed as compensation for the corneal surface increase on inflation test. But the average corneal thickness decreased more than expected theoretically; a small reduction in corneal volume did take place during the pressure increase [29]. The modified Scheimpflug device can provide the data of corneal volume in different range from the apex of the cornea and may benefit investigating the interaction of fluid flows within the stroma and corneal load. In the experiment, our data showed that the corneal volume decreased by approximately $7.32 \%$ for a pressure increase from $15 \mathrm{mmHg}$ to $75 \mathrm{mmHg}$ and it further supported the idea that local fluid shifts in the stroma are possible within minutes after load changes.

Young's modulus is a measure of property of elastic materials and it is defined as the ratio between stress and strain. Mammalian corneas demonstrated hyperelastic behavior with an initial low stiffness and a final high stiffness under short-term inflation testing. The stress-strain relationship of the cornea is not linear. Therefore, it is inappropriate to use Young's elastic modulus to describe the nonlinear biomechanical properties of the cornea [30]. In this study, bulk modulus defined as the ratio of the infinitesimal pressure increase to the resulting relative decrease of the volume was introduced to describe the corneal biomechanics. The changes of the corneal bulk modulus were investigated with 
the posterior pressure increase and we found that the bulk modulus decreased gradually to the platform. It suggested that the cornea was almost viewed as an incompressible soft tissue especially with the stress of more high posterior pressure.

In conclusion, the modified Scheimpflug device is a valuable method to measure the parameters of anterior segment on the inflation tests and has more advantages of the measurements of biomechanics of the cornea. The variations of the corneal biomechanics due to the refractive corneal surgeries or corneal transplantation may be further investigated by this device.

\section{Competing Interests}

The authors state that they have no commercial or proprietary interest in the products named in the paper.

\section{Acknowledgments}

This study was supported by Guangdong Science and Technology Project Fund (2002C30902).

\section{References}

[1] J. Liu and C. J. Roberts, "Influence of corneal biomechanical properties on intraocular pressure measurement: quantitative analysis," Journal of Cataract and Refractive Surgery, vol. 31, no. 1, pp. 146-155, 2005.

[2] T. T. Andreassen, A. H. Simonsen, and H. Oxlund, "Biomechanical properties of keratoconus and normal corneas," Experimental Eye Research, vol. 31, no. 4, pp. 435-441, 1980.

[3] V. Alastrué, B. Calvo, E. Peña, and M. Doblaré, "Biomechanical modeling of refractive corneal surgery," Journal of Biomechanical Engineering, vol. 128, no. 1, pp. 150-160, 2006.

[4] D. A. Hoeltzel, P. Altman, K. Buzard, and K.-I. Choe, "Strip extensiometry for comparison of the mechanical response of bovine, rabbit, and human corneas," Journal of Biomechanical Engineering, vol. 114, no. 2, pp. 202-215, 1992.

[5] A. Elsheikh, D. Alhasso, and P. Rama, "Biomechanical properties of human and porcine corneas," Experimental Eye Research, vol. 86, no. 5, pp. 783-790, 2008.

[6] A. Elsheikh and D. Wang, "Numerical modelling of corneal biomechanical behaviour," Computer Methods in Biomechanics and Biomedical Engineering, vol. 10, no. 2, pp. 85-95, 2007.

[7] A. Elsheikh and K. Anderson, "Comparative study of corneal strip extensometry and inflation tests," Journal of the Royal Society Interface, vol. 2, no. 3, pp. 177-185, 2005.

[8] J. O. Hjortdal, "Biomechanical studies of the human cornea: development and application of a method for experimental studies of the extensivility of the intact human cornea," Acta Ophthalmologica Scandinavica, vol. 73, no. 4, pp. 364-365, 1995.

[9] S. L.-Y. Woo, A. S. Kobayashi, W. A. Schlegel, and C. Lawrence, "Nonlinear material properties of intact cornea and sclera," Experimental Eye Research, vol. 14, no. 1, pp. 29-39, 1972.

[10] P. D. Jaycock, L. Lobo, J. Ibrahim, J. Tyrer, and J. Marshall, "Interferometric technique to measure biomechanical changes in the cornea induced by refractive surgery," Journal of Cataract and Refractive Surgery, vol. 31, no. 1, pp. 175-184, 2005.
[11] Ö. Ö. Uçakhan, M. Özkan, and A. Kanpolat, "Corneal thickness measurements in normal and keratoconic eyes: pentacam comprehensive eye scanner versus noncontact specular microscopy and ultrasound pachymetry," Journal of Cataract and Refractive Surgery, vol. 32, no. 6, pp. 970-977, 2006.

[12] U. de Sanctis, A. Missolungi, B. Mutani, L. Richiardi, and F. M. Grignolo, "Reproducibility and repeatability of central corneal thickness measurement in keratoconus using the rotating Scheimpflug camera and ultrasound pachymetry," The American Journal of Ophthalmology, vol. 144, no. 5, pp. 712718.e1, 2007.

[13] D. Chen and A. K. C. Lam, "Reliability and repeatability of the Pentacam on corneal curvatures," Clinical and Experimental Optometry, vol. 92, no. 2, pp. 110-118, 2009.

[14] D. P. Piñero, C. Saenz González, and J. L. Alió, "Intraobserver and interobserver repeatability of curvature and aberrometric measurements of the posterior corneal surface in normal eyes using Scheimpflug photography," Journal of Cataract and Refractive Surgery, vol. 35, no. 1, pp. 113-120, 2009.

[15] D. Chen and A. K. C. Lam, "Intrasession and intersession repeatability of the Pentacam system on posterior corneal assessment in the normal human eye," Journal of Cataract and Refractive Surgery, vol. 33, no. 3, pp. 448-454, 2007.

[16] C. A. Utine, F. Altin, H. Cakir, and I. Perente, "Comparison of anterior chamber depth measurements taken with the Pentacam, Orbscan IIz and IOLMaster in myopic and emmetropic eyes," Acta Ophthalmologica, vol. 87, no. 4, pp. 386-391, 2009.

[17] J.-H. Yi, S. Hong, G. J. Seong, S. Y. Kang, K. T. Ma, and C. Y. Kim, "Anterior chamber measurements by pentacam and AS-OCT in eyes with normal open angles," Korean Journal of Ophthalmology, vol. 22, no. 4, pp. 242-245, 2008.

[18] M. A. Miranda, C. O’Donnell, and H. Radhakrishnan, "Repeatability of corneal and ocular aberration measurements and changes in aberrations over one week," Clinical and Experimental Optometry, vol. 92, no. 3, pp. 253-266, 2009.

[19] H. Shankar, D. Taranath, C. T. Santhirathelagan, and K. Pesudovs, "Repeatability of corneal first-surface wavefront aberrations measured with Pentacam corneal topography," Journal of Cataract and Refractive Surgery, vol. 34, no. 5, pp. 727-734, 2008.

[20] J.-D. Ho, C.-Y. Tsai, R. J.-F. Tsai, L.-L. Kuo, I.-L. Tsai, and S.W. Liou, "Validity of the keratometric index: evaluation by the Pentacam rotating Scheimpflug camera," Journal of Cataract and Refractive Surgery, vol. 34, no. 1, pp. 137-145, 2008.

[21] S. Doganay, P. Bozgul Firat, S. Emre, and S. Yologlu, "Evaluation of anterior segment parameter changes using the Pentacam after uneventful phacoemulsification," Acta Ophthalmologica, vol. 88, no. 5, pp. 601-606, 2010.

[22] A. Wegener and H. Laser-junga, "Photography of the anterior eye segment according to Scheimpflug's principle: options and limitations-a review," Clinical and Experimental Ophthalmology, vol. 37, no. 1, pp. 144-154, 2009.

[23] H. Hennighausen, S. T. Feldman, J. F. Bille, and A. D. McCulloch, "Anterior-posterior strain variation in normally hydrated and swollen rabbit cornea," Investigative Ophthalmology and Visual Science, vol. 39, no. 2, pp. 253-262, 1998.

[24] D. H. Glass, C. J. Roberts, A. S. Litsky, and P. A. Weber, "A viscoelastic biomechanical model of the cornea describing the effect of viscosity and elasticity on hysteresis," Investigative Ophthalmology and Visual Science, vol. 49, no. 9, pp. 3919-3926, 2008. 
[25] D. P. Piñero and N. Alcón, "Corneal biomechanics: a review," Clinical and Experimental Optometry, vol. 98, no. 2, pp. 107-116, 2015.

[26] A. K. C. Lam, Y. Hon, L. K. K. Leung, and D. C. C. Lam, "Repeatability of a novel corneal indentation device for corneal biomechanical measurement," Ophthalmic and Physiological Optics, vol. 35, no. 4, pp. 455-461, 2015.

[27] N. Ehlers, "The fibrillary texture and the hydration of the cornea," Acta Ophthalmologica, vol. 44, no. 4, pp. 620-630, 1966.

[28] B. Jue and D. M. Maurice, "The mechanical properties of the rabbit and human cornea," Journal of Biomechanics, vol. 19, no. 10, pp. 847-853, 1986.

[29] J. O. Hjortdal, "Extensibility of the normo-hydrated human cornea," Acta Ophthalmologica Scandinavica, vol. 73, no. 1, pp. 12-17, 1995.

[30] B. L. Boyce, R. E. Jones, T. D. Nguyen, and J. M. Grazier, "Stresscontrolled viscoelastic tensile response of bovine cornea," Journal of Biomechanics, vol. 40, no. 11, pp. 2367-2376, 2007. 


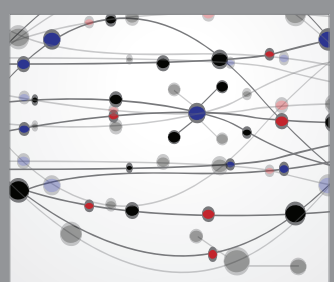

The Scientific World Journal
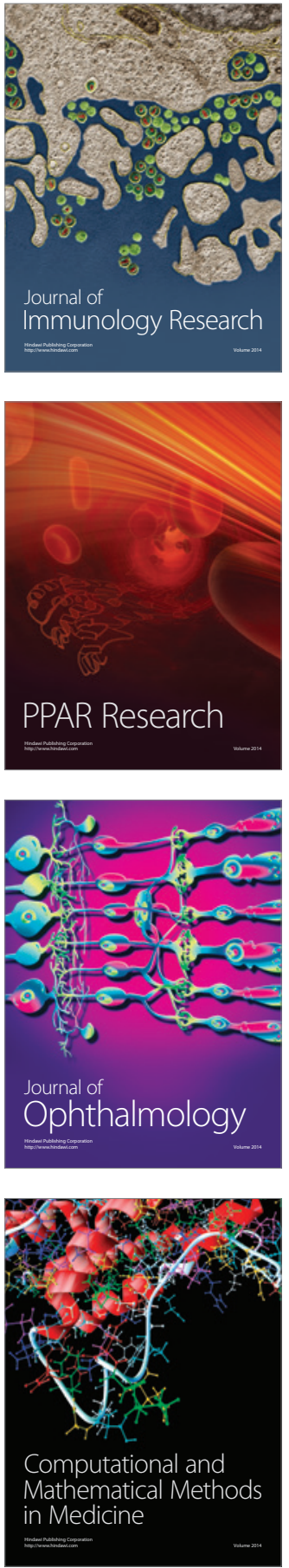

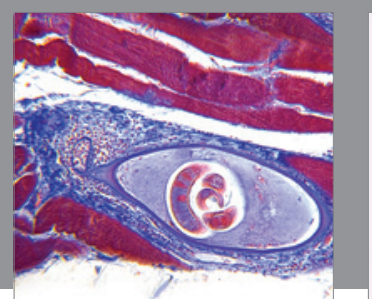

Gastroenterology Research and Practice

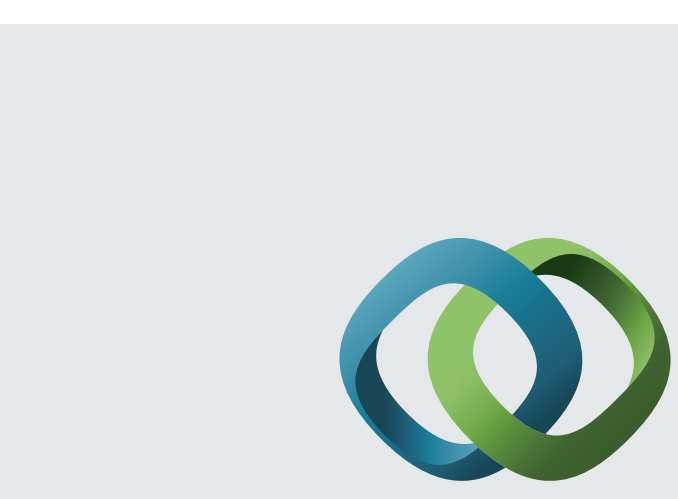

\section{Hindawi}

Submit your manuscripts at

http://www.hindawi.com
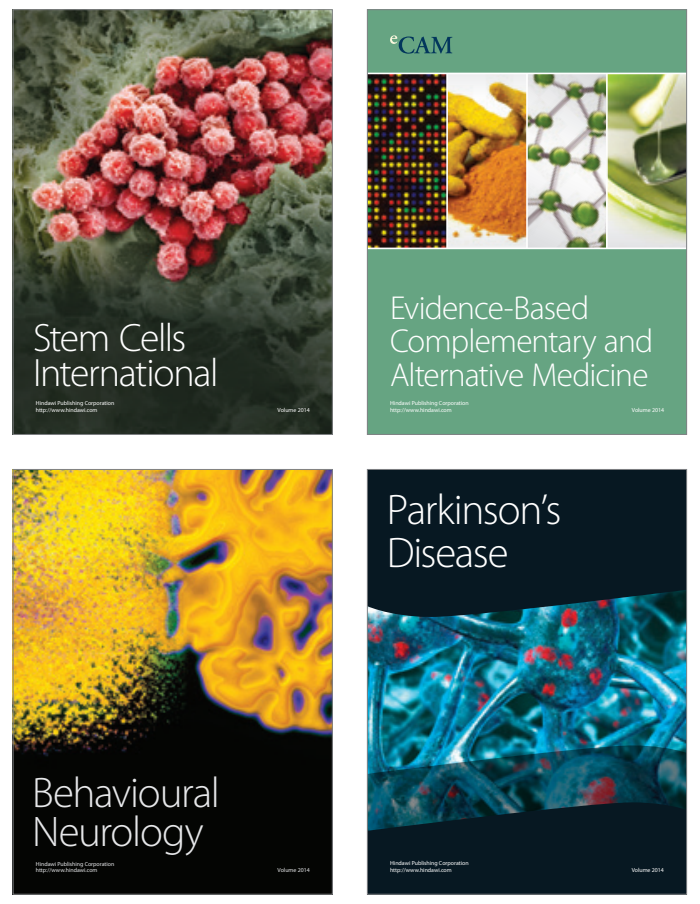
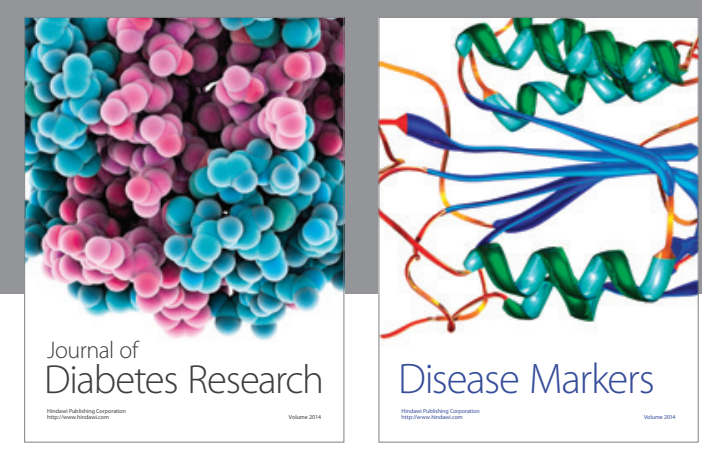

Disease Markers
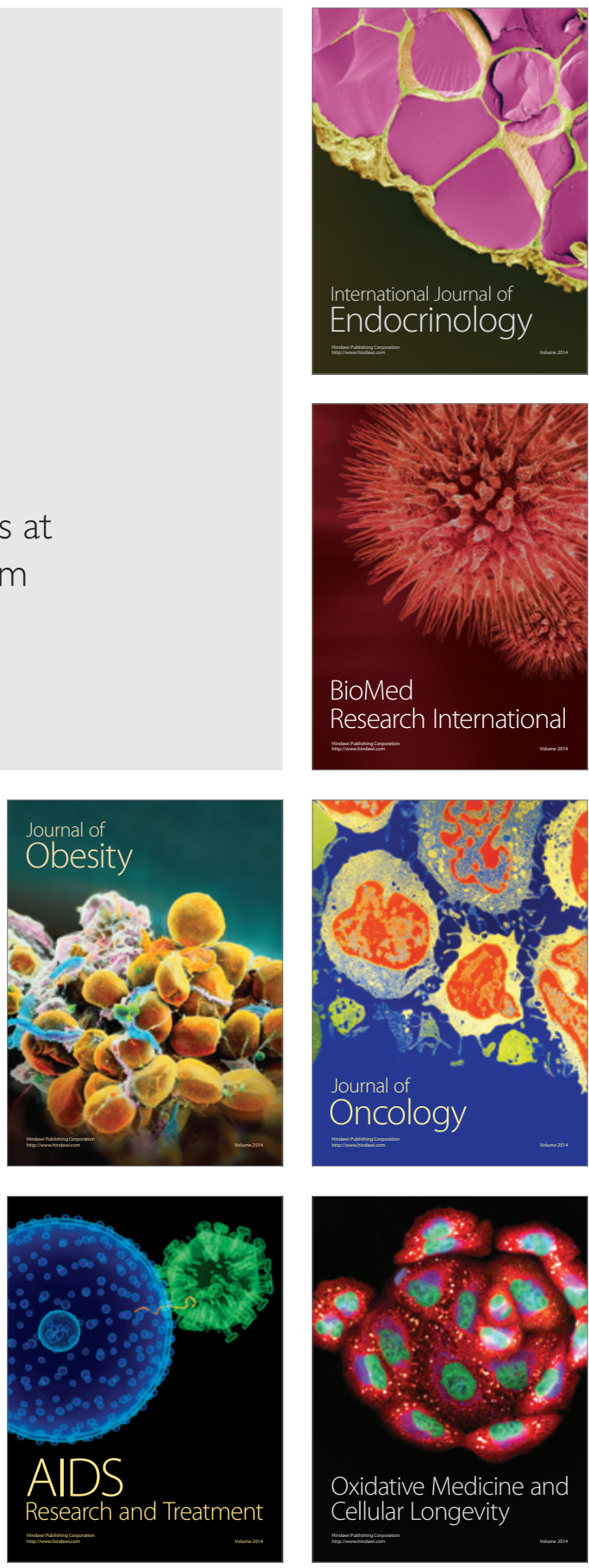